\title{
La consistencia en la interfaz de usuario
}

UNO DE LOS ASPECTOS MÁS RELEVANTES de la usabilidad de un sistema es la consistencia de su interfaz de usuario. Aunque como objetivo general el término consistencia hace referencia a secuencia de acciones, términos, unidades, colores o tipografía comunes dentro de un mismo programa de aplicación o un mismo escenario, la consistencia no tiene significado en sí misma, es inherentemente un concepto relacional.

\section{Componentes de la consistencia}

Wendy Kellogg (2001) sugiere dos componentes fundamentales en la consistencia:

La componente descriptiva en la que la caracterización de la interfaz es elaborada en términos de las propiedades para las que es o no consistente. Por ejemplo: aspecto visual, objetos referentes del mundo real o el modelo mental.

La componente evaluativa plantea la decisión acerca de la bondad del diseño en relación con el uso para el que supuestamente está destinado.

En la descripción de la consistencia de una interfaz siempre acecha el peligro de quedarse en un primer nivel, limitándose sólo a las características más tangibles (apariencia estética o comportamiento). Sin embargo, inclinar la balanza

¿Recibes ya EPI en casa?

Por sólo $65 \not a+4 \%$ IVA puedes tener todos los meses tu copia particular y leerla cómodamente cuando quieras.

\section{Por Antonio Moreno Muñoz}

hacia la evaluación y elección entre distintas alternativas potenciales de diseño, aportará los beneficios de una completa articulación de las dimensiones de la consistencia. Para ello acudiremos a la inspección de consistencia.

http://www.acm.org/sigchi/chi95/p roceedings/tutors/jn_bdy.htm

http://www.entrelinea.com/usabili dad/inspeccion/Consistency.htm

\section{Dimensiones de la consistencia}

Es posible analizar la consistencia a diferentes niveles: en una aplicación individual, en una familia de productos, para todos los productos distribuidos por el mismo comerciante, para todos los productos que trabajan en un mismo sistema operativo, nacional, comunitario, internacional.

$$
\begin{aligned}
& \text { «En la descrip- } \\
& \text { ción de la con- } \\
& \text { sistencia de una } \\
& \text { interfaz siempre } \\
& \text { acecha el peli- } \\
& \text { gro de quedarse } \\
& \text { en un primer ni- } \\
& \text { vel, limitándose } \\
& \text { sólo a las carac- } \\
& \text { terísticas más } \\
& \text { tangibles (apa- } \\
& \text { riencia estética } \\
& \text { o comporta- } \\
& \text { miento)» }
\end{aligned}
$$

Las dos primeras son denominadas consistencia interna y exter$n a$ respectivamente. $\mathrm{Y}$ suelen ser las que mayores desvelos originan en los fabricantes de programas informáticos. En la consistencia externa también se estudian las relaciones con el mundo real bajo el término: consistencia física. http://www.asktog.com/basics/first Principles.html

http://cfg.cit.cornell.edu/cfg/desig $n /$ concepts.html\#consistency

\section{Metodologías de trabajo}

Existe un abanico suficiente de metodologías para el aseguramiento de la consistencia (Jacob Nielsen, 2001). Bruce Tognazzini (2001) nos revela en un artículo la importancia de la consistencia en el éxito del Macintosh de Apple. Es necesario apuntar que, en el seguimiento de unas directivas para la accesibilidad del sistema, no se trata de normativa de obligado cumplimiento, sino que se habla generalmente de recomendaciones. Es necesario un posicionamiento más flexible, de manera que no se pueda coartar la creatividad del diseñador. En este sentido es muy esclarecedor el artículo de Liang-Yi Lin y James A. Levin. Pero para terminar, atención, como en cualquier proceso de implantación de la calidad, el reto debe ser asumido por todo el equipo de trabajo y toda la empresa.

http://www.ed.uiuc.edu/tta/papers/ lin-levin/

\section{Bibliografía}

Kellogg, Wendy. "The dimensions of consistency". En: Coordinating user interfaces for consistency. Jakob Nielsen (ed.). Boston: Academic Press, 2001.

Nielsen, Jakob. "Executive summary: coordinating user interfaces for consistency". En: $\mathrm{Co}$ ordinating user interfaces for consistency. Jakob Nielsen (ed.). Boston: Academic Press, 2001.

Tognazzini, Bruce (2001). “Achieving consistency for the macintosh". En: Coordinating user interfaces for consistency. Jakob Nielsen (ed.). Boston: Academic Press, 2001.

Antonio Moreno Muñoz

ellmomua@uco.es

http://www.uco.es/ ellmomual

ergonomial

Universidad de Córdoba 